OPEN ACCESS

Edited by:

Fu Wang,

Xi'an Jiaotong University, China

Reviewed by:

Laurent Metzinger,

University of Picardie Jules

Verne, France

Yanqing Liu,

Columbia University, United States

${ }^{*}$ Correspondence:

Nianhan Ma

nianhan.ma@g.ncu.edu.tw

Chiu-Ching Huang

drcchhuang@gmail.com

Specialty section: This article was submitted to

Precision Medicine,

a section of the journal

Frontiers in Medicine

Received: 16 June 2021 Accepted: 07 September 2021

Published: 01 October 2021

Citation:

Li A-L, Chou C-Y, Chen C-L, Wu K-L,

Lin S-C, Chen H-C, Wang M-C,

Chang C-C, Hsu B-G, Wu M-S, MaN and Huang C-C (2021) The MicroRNA

Prediction Models as Ancillary

Diagnosis Biomarkers for Urothelial

Carcinoma in Patients With Chronic

Kidney Disease.

Front. Med. 8:726214.

doi: 10.3389/fmed.2021.726214

\section{The MicroRNA Prediction Models as Ancillary Diagnosis Biomarkers for Urothelial Carcinoma in Patients With Chronic Kidney Disease}

\author{
An-Lun Li ${ }^{1}$, Che-Yi Chou ${ }^{2}$, Chien-Lung Chen ${ }^{3}$, Kun-Lin Wu ${ }^{1,4}$, Shih-Chieh Lin ${ }^{5}$, \\ Hung-Chun Chen ${ }^{6}$, Ming-Cheng Wang ${ }^{7}$, Chia-Chu Chang ${ }^{8,9}$, Bang-Gee Hsu ${ }^{10}$, \\ Mai-Szu Wu ${ }^{11}$, Nianhan Ma ${ }^{1 \star}$ and Chiu-Ching Huang ${ }^{12 \star}$
}

\footnotetext{
${ }^{1}$ Department of Biomedical Sciences and Engineering, National Central University, Taoyuan, Taiwan, ${ }^{2}$ Division of Nephrology, Department of Internal Medicine, Asia University Hospital, Taichung, Taiwan, ${ }^{3}$ Department of Nephrology, Landseed International Hospital, Taoyuan, Taiwan, ${ }^{4}$ Division of Nephrology, Department of Internal Medicine, Taoyuan Armed Forces General Hospital, Taoyuan, Taiwan, ${ }^{5}$ Institute of Basic Medical Sciences, College of Medicine, National Cheng Kung University, Tainan, Taiwan, ${ }^{6}$ Division of Nephrology, Kaohsiung Medical University, Kaohsiung, Taiwan, ${ }^{7}$ Division of Nephrology, Cheng Kung University Hospital, Tainan, Taiwan, ${ }^{8}$ Division of Nephrology, Department of Internal Medicine, Kuang Tien General Hospital, Taichung, Taiwan, ${ }^{9}$ Department of Nutrition, Hungkuang University, Taichung, Taiwan, ${ }^{10}$ Division of Nephrology, Buddhist Tzu Chi General Hospital, Hualien, Taiwan, ${ }^{11}$ Division of Nephrology, Taipei Medical University and Hospitals, Taipei, Taiwan, ${ }^{12}$ Division of Nephrology and The Kidney Institute, China Medical University and Hospitals, Taichung, Taiwan
}

Urothelial carcinoma is a common urological cancer in chronic kidney disease patients. Cystoscopy and urine cytology are the clinical diagnostic tools for UC. However, cystoscopy is an invasive procedure, while urine cytology showed low sensitivity for low-grade urothelial tumors. High accuracy with non-invasive tools for UC is needed for CKD patients. Our study collected a total of 272 urine and 138 plasma samples to detect the miRNA expression levels for establishing UC signatures from CKD patients. Seventeen candidate miRNAs of biofluids were selected and confirmed by qRT-PCR. Our results showed that urinary miR-1274a and miR-30a-5p expression levels were significantly lower but miR-19a-5p expression levels were higher in UC when compared with CKD. In plasma samples, miR-155-5p, miR-19b-1-5p, miR-378, and miR-636 showed significantly lower expression in UC compared to those with CKD. The Kaplan-Meier curve showed that lower expression of miR-19a, miR-19b, miR-636 and miR-378, and higher expression of miR-708-5p were associated with poor prognosis in patients with bladder cancer. In addition, we produced classifiers for predicting UC by multiple logistic regression. The urine signature was developed with four miRNAs, and the AUC was 0.8211. Eight miRNA expression levels from both urine and plasma samples were examined, and the AUC was 0.8595. Two miRNA classifiers and the nomograms could improve the drawbacks of current UC biomarker screenings for patients with CKD.

Keywords: microRNA (miRNA), urothelial carcinoma (UC), chronic kidney disease, biomarker, biofluid 


\section{INTRODUCTION}

Urothelial carcinoma (UC) includes bladder cancer and urinary tract cancer. A worldwide report revealed $\sim 549,393$ newly diagnosed cases and 199,922 deaths from UC in 2018 (1). UC accounts for $90 \%$ of bladder cancer and is the most common malignancy involving the urinary tract (2). UC is responsible for $31 \%$ of urinary tract cancers in Taiwan (3). $51-58.6 \%$ of UC patients have chronic kidney disease (CKD) and patients with CKD are more at risk of UC (3-6). Advanced stages of CKD are associated with poor prognosis for UC treatment $(7,8)$. Painless hematuria is the most common presenting symptom in UC, but painless hematuria is also common in patients with CKD. The sensitivity and specificity of UC protein markers are decreased because the serum protein levels are increased in patients with CKD (9-13). Intravenous pyelography or urography cannot be performed in patients with CKD because of the exposure of contrast media (14). The high specificity of urinary cytology can be interfered by the presence of CKD (15). Invasive cystoscopy or ureteroscopy are usually needed to confirm the diagnosis of UC. In addition, the cost of cystoscopy or ureteroscopy is expensive, the procedure is invasive and uncomfortable, and patients need to experience the risk of anesthesia and surgery. Therefore, developing highly accurate non-invasive biomarkers for UC is urgently needed for patients with CKD.

The miRNA pattern in biofluids was thought to provide disease molecular markers to predict or differentiate different types of cancers because the development of cancer is associated with the expression levels of circulating miRNAs (16-18). In addition, miRNAs can be packed and released through exosomes or extracellular vesicles, enhancing their stability in biofluids such as urine and plasma. Some reports have discussed the difference in miRNA expression in biofluids for predicting urological tumors, but most of the studies compared healthy donors with patients with cancer $(16,19-23)$. Our previous study demonstrated that the miRNA classifier of plasma predicted UC in patients with ESRD (24). In the present work, we investigated the expression levels of miRNAs in the urine and plasma of patients with CKD. We further used these miRNA signatures to develop prediction models of UC for patients with CKD.

\section{MATERIALS AND METHODS}

\section{Patients and Samples}

The Taiwan Urothelial Cancer Consortium (TUCC) organized a multicenter study of urothelial cancer (UC) from ten hospitals in Taiwan. The ten hospitals are distributed throughout the country (13) (Supplementary Table 1). A total of 272 patients (50, 111, and 111 samples were healthy, CKD and CKD + UC, respectively) participated in this study. The urine and blood samples were collected from control patients after obtaining informed consents. The urine and blood samples were collected from CKD+UC patients within 3 days before the surgery. Samples were centrifuged at 1,700 and 2,000 $\times \mathrm{g}$ for $20 \mathrm{~min}$. The supernatant was collected and stored at $-80^{\circ} \mathrm{C}$.

\section{Ethics Approval and Informed Consent}

This study was approved by the internal review board (IRB) of China Medical University Hospital (CMUH 102-REC2- 043) and the IRB of each hospital. Written informed consent was obtained from all patients to use their urine and blood samples. All methods were followed in accordance with guidelines and regulations.

\section{Total RNA Isolation From Biofluids and miRNA Quantification by RT-PCR}

Total RNA from urine and plasma was extracted using TRIzol ${ }^{\circledR}$ LS Reagent and a mirVana ${ }^{\mathrm{TM}}$ miRNA Isolation Kit according to the standard protocol. The spiked-in control of cel-miR-39-3p for technical variability followed the previously described (24). The RNA quality was detected by a spectrophotometer (BioTek Instruments, Take3 microplate). The ratios of absorbance $260 \mathrm{~nm}$ to the absorbance at 280 or $230 \mathrm{~nm}$ have been used as the reference of the purity of RNA samples $\left(\mathrm{A}_{260} / \mathrm{A}_{280} \fallingdotseq 2, \mathrm{~A}_{260} / \mathrm{A}_{230}\right.$ $\fallingdotseq 2-2.2)$. All RNA samples were stored at $-80^{\circ} \mathrm{C}$. The TaqMan ${ }^{\mathrm{TM}}$ MicroRNA Reverse Transcription Kit (Applied Biosystems) was used to produce the cDNAs from miRNAs, and the standard protocol or ingredients were followed as described previously (25). The microRNA profiling was generated using TaqMan ${ }^{\circledR} 2 \mathrm{x}$ Universal PCR master mix without UNG and TaqMan ${ }^{\circledR}$ Array Human MicroRNA Cards (4444913). TaqMan ${ }^{\circledR}$ miRNA assays quantified the specific miRNAs expression (4427975) (Thermo Fisher Scientific).

\section{Data Statistical Analysis}

The expression of miRNAs was determined using the $2^{-\Delta C T}$ method relative to RNU6. The miRNA expression data were transformed to the $\log _{10}$ form to fit a normal distribution. The value of no detection of miRNA expression was replaced with the -4.5 value in the $\log _{10}$ form. Clinical characteristics between healthy, CKD and CKD+UC patients were evaluated using Pearson's chi-squared test for each variable. Normality and Student's $t$-test were used for unpaired comparisons of two groups. All tests were two-tailed and were assessed by Levene's test. All statistical analyses were completed with GraphPad Prism software. Logistic regression of miRNA expression was combined with SigmaPlot software. All statistic methods or procedures were followed as described previously (24).

\section{Survival Curve Analysis}

A KM-plotter analysis was performed to integrate the miRNA expression and survival data from TCGA, GEO and EGA database (http://kmplot.com/analysis/index.php?p= background). miRNA expression values from clinical specimens were used to perform Kaplan-Meier survival curve analysis according to the clinical parameters provided. High and low expression groups were created using an automatic cutoff as described previously (25). The miRNAs expression associated with UC in multivariable logistic regression was used to generate a nomogram for UC. The coding packages of RMS in R software were used to develop the nomogram of UC. 


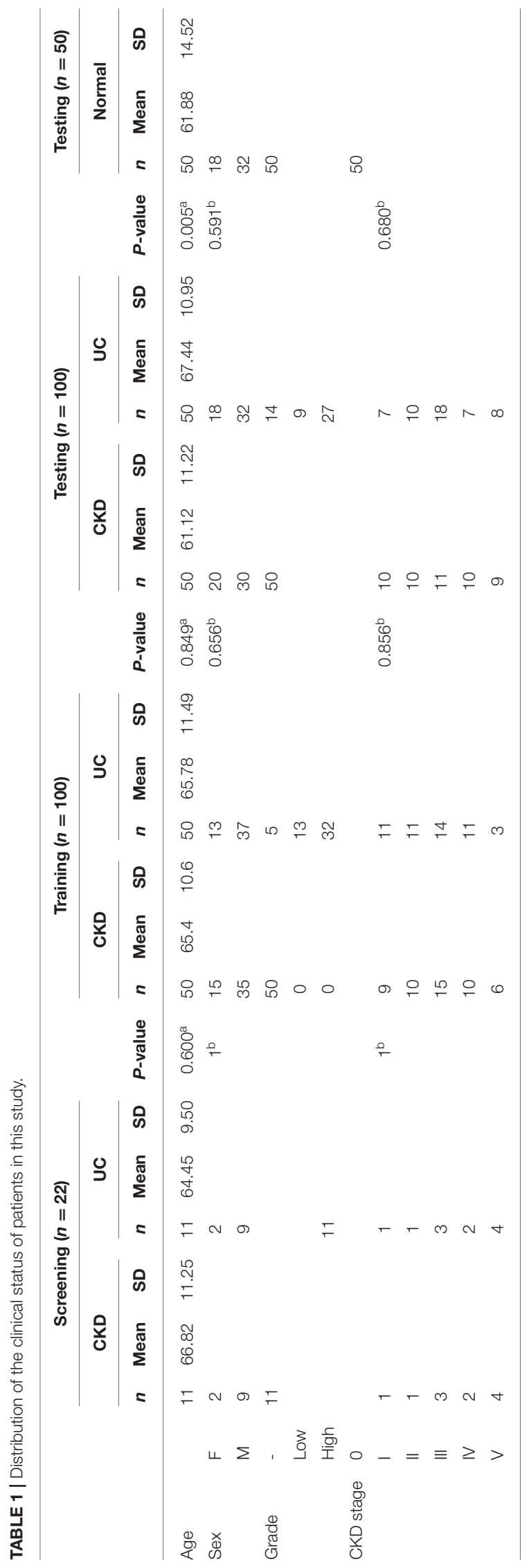

TABLE 2 | miRNA names and sequences.

\begin{tabular}{ll}
\hline miRNA name & Mature miRNA sequence \\
\hline hsa-miR-586 & UAUGCAUUGUAUUUUUAGGUCC \\
hsa-miR-129-5p & CUUUUUGCGGUCUGGGCUUGC \\
hsa-miR-33b-5p & GUGCAUUGCUGUUGCAUUGC \\
hsa-miR-30a-5p & UGUAAACAUCCUCGACUGGAAG \\
hsa-miR-1274A & GUCCCUGUUCAGGCGCCA \\
hsa-miR-126-3p & UCGUACCGUGAGUAAUAUGCG \\
hsa-miR-210-3p & CUGUGCGUGUGACAGCGGCUGA \\
hsa-miR-202-3p & AGAGGUAUAGGGCAUGGGAA \\
hsa-miR-19a-5p & AGUUUUGCAUAGUUGCACUACA \\
hsa-miR-708-5p & AAGGAGCUUACAAUCUAGCUGGG \\
hsa-miR-19b-1-5p & AGUUUUGCAGGUUUGCAUCCAGC \\
hsa-miR-183-3p & GUGAAUUACCGAAGGGCCAUAA \\
hsa-miR-636 & UGUGCUUGCUCGUCCCGCCCGCA \\
hsa-miR-155-5p & UUAAUGCUAAUCGUGAUAGGGGU \\
hsa-miR-378 & ACUGGACUUGGAGUCAGAAGG \\
hsa-miR-487a-3p & AAUCAUACAGGGACAUCCAGUU \\
hsa-miR-150-5p & UCUCCCAACCCUUGUACCAGUG \\
\hline
\end{tabular}

\section{RESULTS}

\section{Differentially Expressed Urine and Plasma miRNAs Between CKD and CKD + UC}

In order to discover an ancillary diagnostic tool for UC in patients with CKD, all samples were collected from ten hospitals throughout Taiwan from 2013 to 2018. We matched the patients with CKD and CKD+UC by sex, age, and CKD stage to select the difference in miRNA expression levels in this study (Table 1). For $\mathrm{CKD}+\mathrm{UC}$, blood and urine samples were collected within 3 days before surgery. For the control group, blood and urine samples were collected after tracking their renal functions as CKD. Next, the high throughput and quantitative real-time miRNA PCR array including 754 miRNAs were utilized to detect 22 (11 CKD and $11 \mathrm{CKD}+\mathrm{UC})$ and $16(8 \mathrm{CKD}$ and $8 \mathrm{CKD}+\mathrm{UC})$ samples of urine and plasma, respectively (Supplementary Table 3). We not only calculated the relative expression levels by RNU6 but also calculated the miRNA ratio of two different miRNAs expression to remove the normalization problem in cell-free biofluids. To date, no literature has noted that any miRNA is a competent internal control in biofluids, and we found that the ratio value method could reduce individual sample differences. We compared miRNA expressions between CKD and CKD + UC samples, and 17 candidate miRNAs were selected from screen set (Table 2).

Next, we validated the expression levels of 17 candidate miRNAs from a screening set by the single qRT-PCR method and measured 200 urine samples (100 CKD and $100 \mathrm{CKD}+\mathrm{UC})$ and 138 plasma samples (74 CKD and $64 \mathrm{CKD}+\mathrm{UC})$ in training and testing set (Supplementary Table 2). Our results showed that the expression of seven miRNAs was significantly different between the CKD and CKD + UC samples (Figure 1). In urine samples, miR-1274a and miR-30a-5p expression levels were significantly decreased ( $p=0.0243$ and 0.0356 , respectively), 
but the miR-19a-5p expression level was significantly increased $(p<0.001)$ (Figure 1A). In the plasma samples, miR-1555p, miR-19b-1-5p, miR-378 and miR-636 expression levels were significantly decreased $(p=0.0324,0.043,0.287$, and 0.0288 , respectively) (Figure 1B). Interestingly, previous study

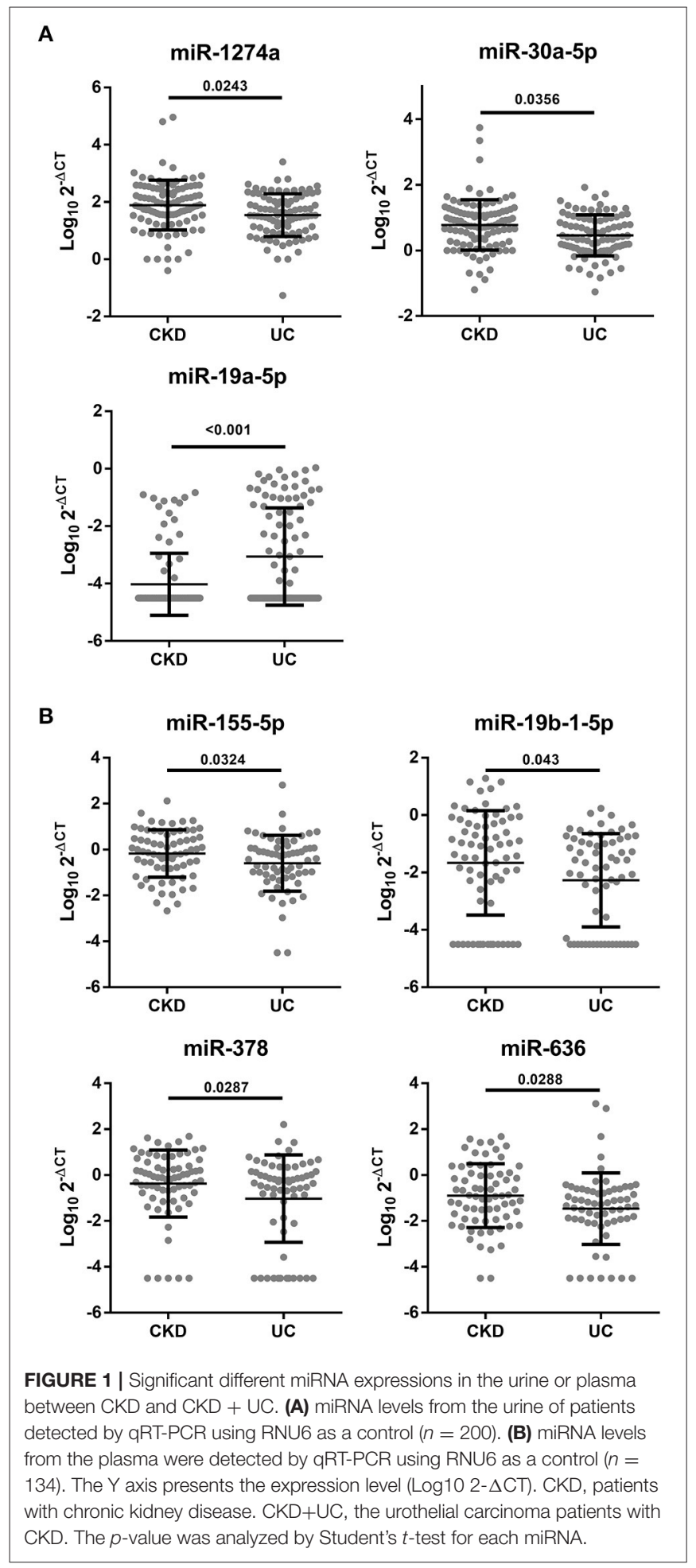

has shown that miR-30a-5p had significantly low expression levels in plasma samples of patients with BC (26). In addition, miR-155-5p expression was also reported to be significantly decreased in the urine sediment cells of patients with BC (27).

Many studies have compared the different miRNA expression levels between the healthy group and patients with UC (28-30). Unlike previous studies, we tried to compare miRNA expression

TABLE 3 | The area under the curve of candidate miRNAs in the training group.

\begin{tabular}{lcc}
\hline Urine sample & AUC & $\mathbf{9 5 \%} \mathbf{C l}$ \\
\hline A & & \\
miR-1274a & 0.71 & $0.6090-0.8110$ \\
miR-19a-5p & 0.61 & $0.4943-0.7169$ \\
miR-30a-5p & 0.64 & $0.5342-0.7514$ \\
miR-708 & 0.63 & $0.6717-0.8611$ \\
\hline Plasma sample & AUC & $95 \%$ Cl \\
\hline B & & \\
miR-155 & 0.65 & $0.5168-0.7773$ \\
miR-19b-1-5p & 0.66 & $0.5327-0.7875$ \\
miR-210-3p & 0.64 & $0.5107-0.7704$ \\
miR-636 & 0.61 & $0.4758-0.7431$ \\
\hline
\end{tabular}

The receiver operating characteristic curve analysis for the candidate miRNAs is shown to distinguish patients with UC from those with CKD through urine samples (A) and plasma samples (B) ( $n=100$ and 70 , respectively).

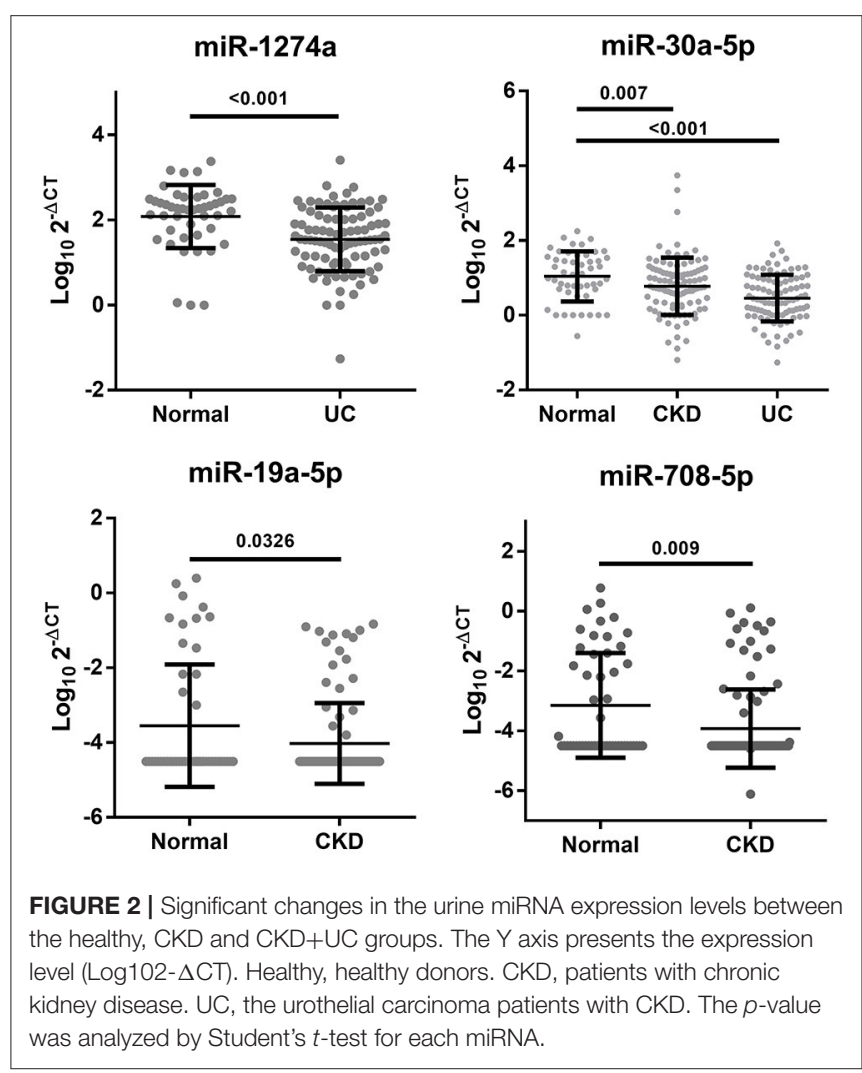


A
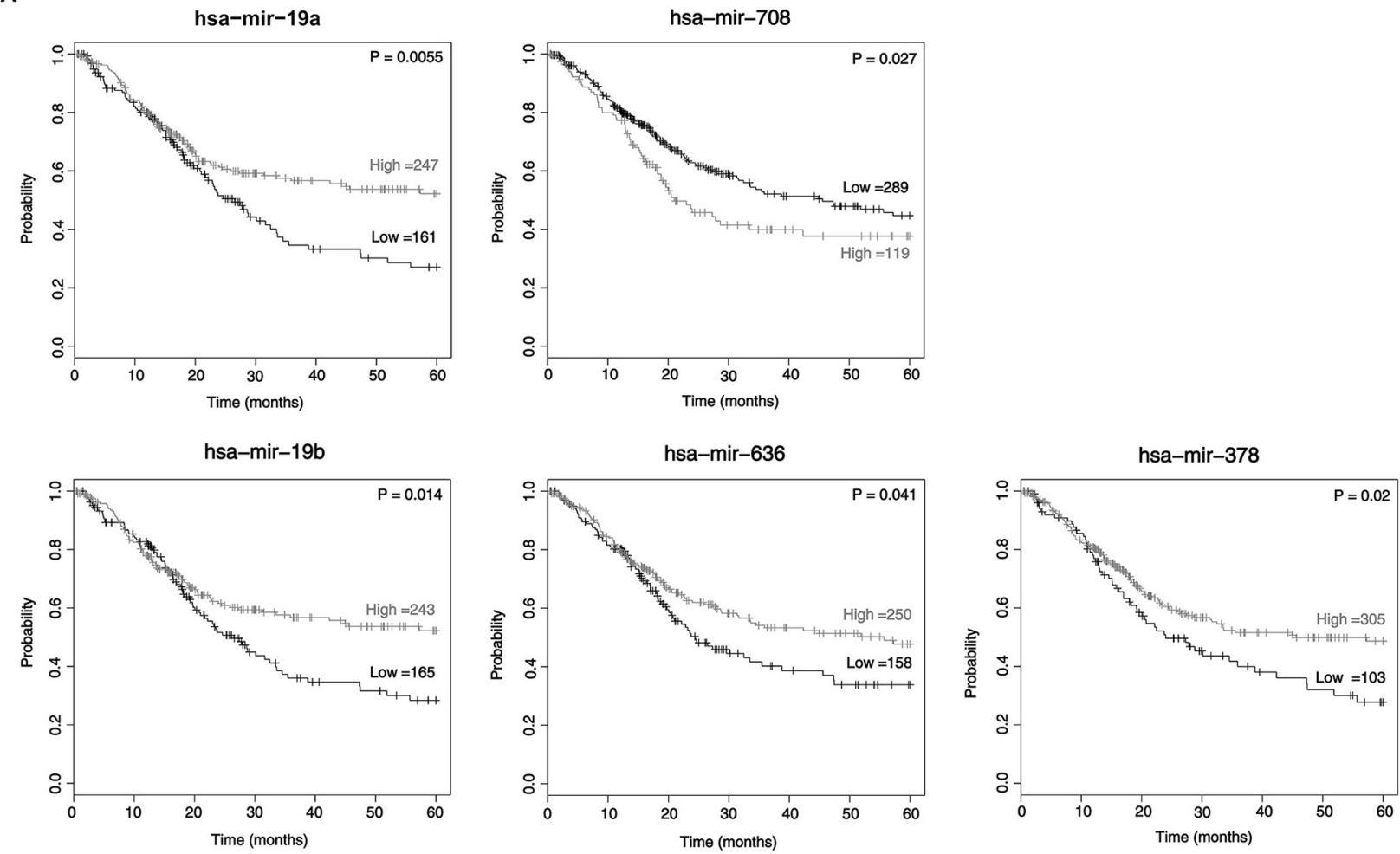

B
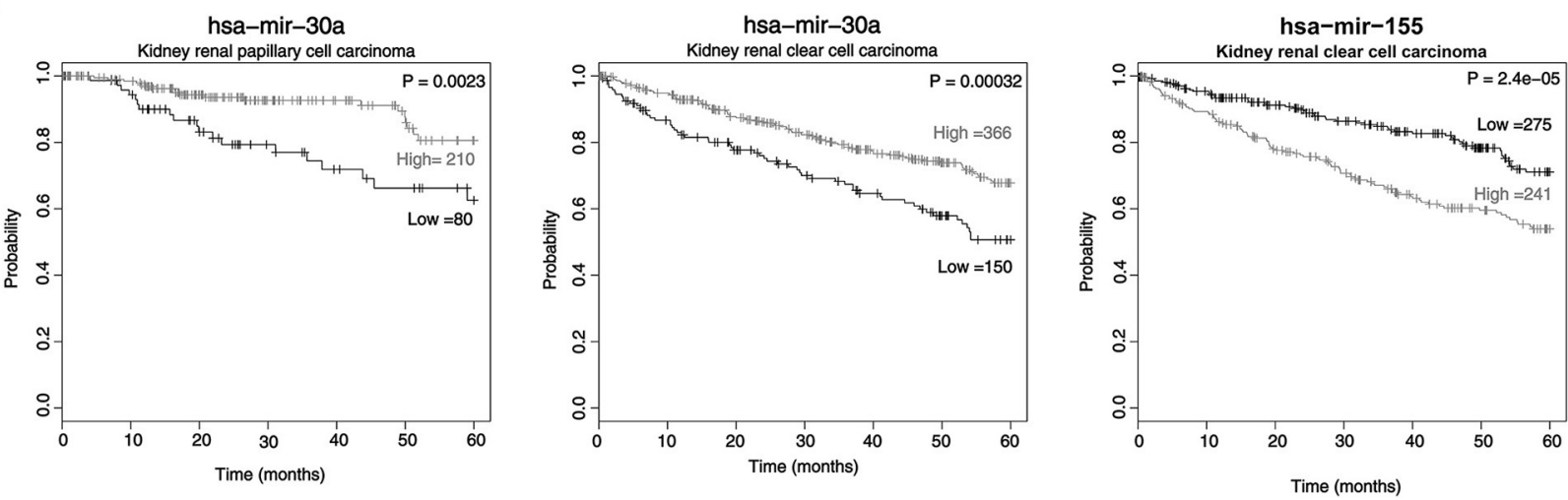

FIGURE 3 | The expression of miRNAs in cancer tissue is associated with survival. The Kaplan-Meier survival curve of patients: low miRNA expression vs. high miRNA expression according to the automatic best cutoff from the database. The statistical significance of the difference in bladder cancer (A) and kidney renal papillary cell carcinoma and kidney renal clear cell carcinoma (B) are shown.

differences to identify UC from patients with CKD. To determine whether these candidate miRNAs from this study also have the potential to distinguish from the healthy group, we further collected 50 healthy cases to analyze the differences within the healthy, CKD and CKD + UC groups. miR-1274a and miR-30a$5 \mathrm{p}$ had significant differences between healthy cases and CKD $+\mathrm{UC}(p<0.001)$. Interestingly, we found that three miRNAs, namely, miR-30a-5p, miR-19a-5p and miR-708-5p, not only can provide a reliable ability to distinguish patients who were $\mathrm{CKD}$ or $\mathrm{CKD}+\mathrm{UC}$ (AUC $=0.64,0.61$, and 0.63 , respectively) but also had significantly different expression levels between healthy subjects and CKD ( $p=0.007,0.0326$, and 0.009 , respectively) (Table 3; Figure 2).

\section{miRNA Expression Levels as a Prognostic Marker of Bladder Cancer and Kidney Cancer}

It has been known that miRNA expression is associated with cancer prognosis. Therefore, we investigated these 17 candidate miRNAs in a public database (http://kmplot.com) to analyze the association between newly identified miRNA expression 
levels and the 5-year survival rate by the Kaplan-Meier method. Among these miRNAs, lower expression levels of miR-19a, miR19b, miR-636, and miR-378 and higher expression levels of miR-708-5p were associated with poor prognosis in BC ( $p=$ $0.0055,0.014,0.041,0.02$, and 0.027, respectively) (Figure 3A). In addition, lower expression of miR-30a and or higher miR155 was associated with poor prognosis in urinary cancer, such as papillary cell carcinoma and clear cell renal cell carcinoma (Figure 3B).

\section{The Prediction Models to Predict UC for Patients With CKD}

To develop a miRNA signature-based predicative model for $\mathrm{UC}$ of patients with all stages of $\mathrm{CKD}$, receiver operating characteristic curve (ROC) analysis was performed. Seventeen candidate miRNA expression levels in urine or plasma from the training set samples were examined. The area under the receiver operating characteristic curve (AUC) is the most commonly used performance measure to indicate the discriminative ability of a prediction mode, and an AUC value higher than 0.6 could be a potential marker. Four miRNAs expressed in urine and four miRNAs expressed in plasma had AUC values above 0.6. The AUC values of miR-1274a, miR-19a-5p, miR-30a-5p and miR$708-5 p$ in urine were $0.71,0.61,0.64$, and 0.628 , respectively $(95 \%$ confidence intervals: $0.6113-0.8198,0.5016-0.7304,0.5980-$ 0.8073 , and $0.5136-0.7424$, respectively) (Table 3A). In plasma samples, miR-155-5p, miR-19b-1-5p, miR-210 and miR-636 could be potential markers, and their AUC values were 0.65, $0.66,0.64$, and 0.61 , respectively (95\% confidence intervals: $0.5168-0.7773,0.5327-0.7875,0.5107-0.7704$, and $0.4758-$ 0.7431, respectively) (Table 3B). Interestingly, these miRNAs have been reported in previous studies to play key functions not only in BC but also in clear cell renal cell carcinoma (31-34).

The combination of multiple factors compared to a single factor always presents more reliable prediction results for clinical classification. Therefore, we utilized multiple logistic regression calculation formulas to produce the prediction model combining different miRNA expression levels from the training group (Table 1). In the urine sample, the top four AUC values for miR-1274A, miR-30a-5p, miR-19b-3p, and miR-708-5p were combined and calculated together, and the AUC was 0.8211 (95\% confidence interval: $0.7359-0.9063)$. We also validated this panel in the testing group, and the data from 200 patients show that the accuracy of the 4-miRNA signature in urine was $70 \%$, based on the cutoff value $>0.483$ (Figure 4A). Furthermore, we added another four miRNAs, namely, miR-155-5p, miR-19b-1-5p, miR210 and miR-636, in plasma to increase the AUC value, and the AUC value increased up to 0.8507 (95\% confidence interval: $0.7751-0.9439)$. The accuracy of the 8 -miRNA signature was $72 \%$, based on the cutoff value $>-0.5940$ (Figure $4 B$ ).

\section{Nomogram Construction Based on miRNAs Expression Signature}

In order to validate the risk of UC, a nomogram integrated miRNAs expression signature was established. The miRNA expression level was transformed to the points based on the cutoff
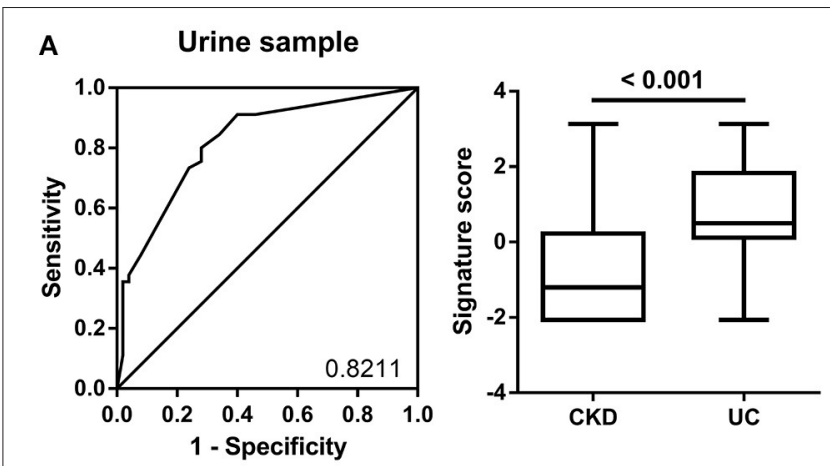

\section{B Urine and Plasma}
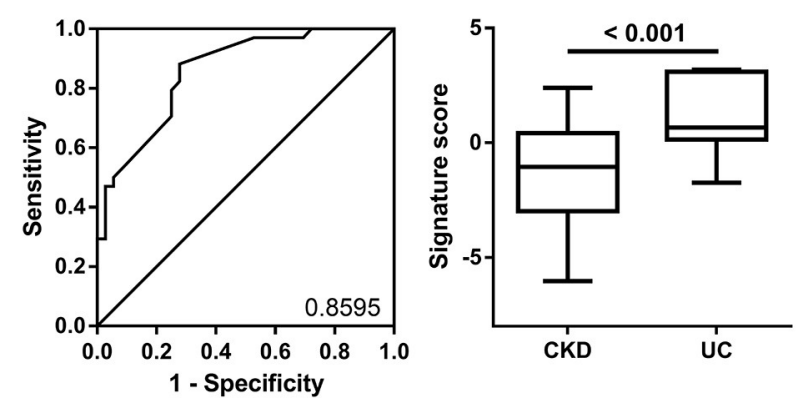

FIGURE 4 | ROC curve analysis of miRNA combinations. (A) ROC curve analysis for miR-1274a, miR-19a-5p, miR-30a-5p, and miR-708-5p in urine was shown to distinguish patients with CKD + UC from those with CKD. (B) The ROC analysis for eight miRNAs (4 in urine-miR-1274a, miR-19a-5p, miR-30a-5p and miR-708-5p, and 4 in plasma-miR-155-5p, miR-19b-1-5p, miR-210 and miR-636) was shown to distinguish patients with CKD+UC from those with CKD. The box plots show the two prediction models distribution that combine the miRNA expression levels from the training group.

value from the training group. The cutoff of miR-1274a, miR-19a5p, miR-30a-5p and miR-708-5p were $<34.41,>2.24^{*} 10^{-4},<$ 3.798 and $>2.235^{*} 10^{-7}$, respectively. The AUC of the nomogram for urine samples were $0.7383(n=200,95 \%$ confidence interval: to $0.6685-0.8080$ ) (Figure 5A). Furthermore, the cutoff of miR155-5p, miR-19b-5p, miR-210-3p, miR-378 and miR-636 were $<1.21,<0.5107,<4.766$, and $<0.5722$, respectively. The AUC of the nomogram for urine and plasma samples were $0.8096(n=$ 138, 95\% confidence interval: 0.7365-0.8827) (Figure 5B).

\section{DISCUSSION}

We found that the expression levels of miR-1274a and miR$30 a-5 p$ were significantly lower in CKD+UC compared with patients with CKD in the urine samples, but conversely, miR-19a$5 p$ was significantly higher in CKD+UC patients (Figure 1A). High expression levels of miR-1274a have been demonstrated in clear cell renal cell carcinoma (ccRCC) compared with adjacent normal cells, which further induced cell apoptosis through the regulation of BMPR1B expression (34). The expression level of miR-30a-5p had significant decreased about $40 \%$ in the plasma samples of the BC patients when compared to the healthy, indicating lower expression of miR-30a-5p in urine due to the 


\section{A}

Points

miR-1274a

miR-19a-5p

miR-30a-5p

miR-708-5p
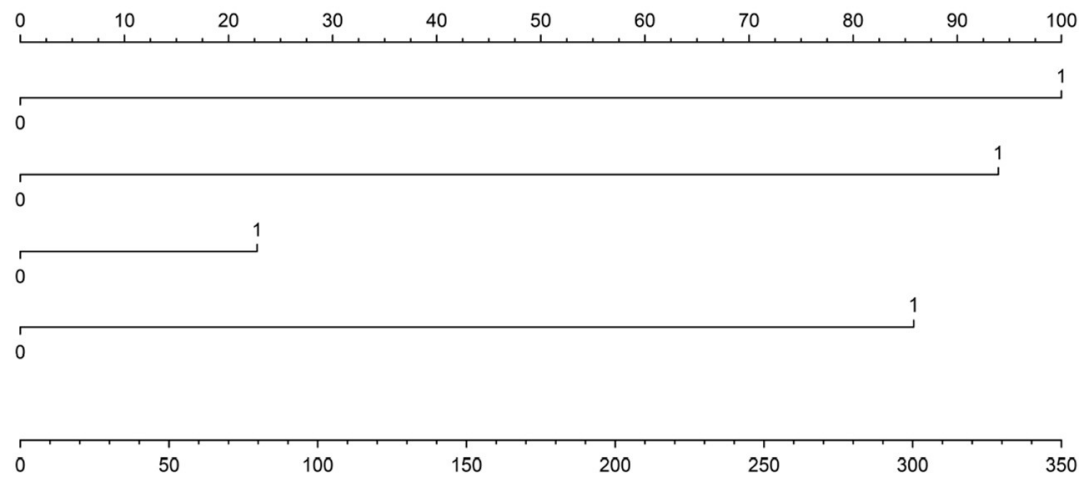

Risk of UC

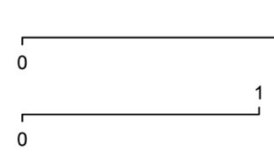

0

Total Points

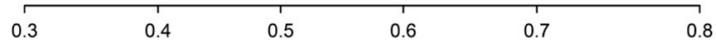

B

Points

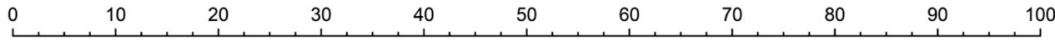

$\operatorname{miR}-1274 a$

$\operatorname{miR}-19 a-5 p$

miR-30a-5p

miR-708-5p

miR-155-5p

miR-19b-5p

miR-210-3p

miR-636

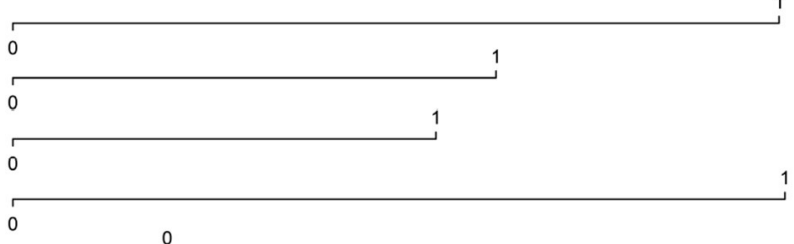

1

1

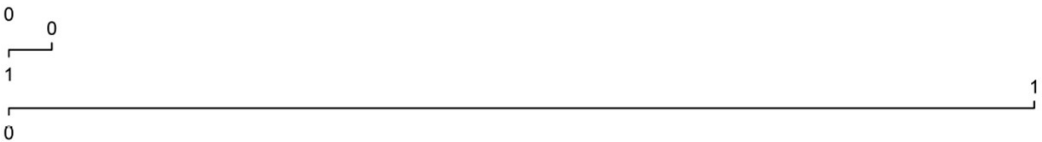

Total Points

Risk of UC

\begin{tabular}{rrrrrrrrrrrr}
\hline & 50 & 100 & 150 & 200 & 250 & 300 & 350 & 400 & 450 & 500 \\
& 0.05 & 0.1 & 0.2 & 0.3 & 0.4 & 0.5 & 0.6 & 0.7 & 0.8 & 0.9 & 0.95
\end{tabular}

FIGURE 5 | Nomogram for the diagnosis of urothelial cancer. (A) Nomogram plot from the points of four miRNA expressions in urine sample. (B) Nomogram plot from the points of eight miRNA expressions in urine and plasma sample.

filtering on renal corpuscle (26). miR-30a-5p showed lower expression in UTUC compared with normal tissue, which was linked to decreased epithelial-to-mesenchymal transition (EMT) through regulation of the tight junction protein claudin-5 (33). Another study showed that miR-30a-5p expression was lower in muscle invasive $B C$ and that overexpression of miR-30a-5p inhibited the malignancy of UC through Notch-1 gene regulation (32). In addition, compared with the healthy group, miR-19a (miR-19a-3p or miR-19a-5p) showed higher expression levels in the samples of BC such as cell lines, tissue and plasma (28).

Our results showed that the expression levels of miR-19b-15p, miR-378, miR-636 and miR-155-5p were significantly lower in $\mathrm{CKD}+\mathrm{UC}$ plasma samples (Figure 1B). The data of hazard ratio showed that the miR-19b (miR-19b-3p or miR-19b-5p) expression level was highly correlated with the incidence of BC. Higher miR-19b (miR-19b-3p or miR-19b-5p) expression levels were found in ccRCC tissue, and miR-19b-3p promoted the malignancy of ccRCC through RhoB gene expression $(35,36)$. Lower miR-378 (miR-378-3p or miR-378-5p) expression levels were significantly linked to the high-risk group suffering from prostate cancer (37). miR-155-3p and miR-155-5p showed a higher expression level in the urine and tissue of patients with $\mathrm{BC}$ $(27,38,39)$. It has been demonstrated that miR-155-5p is a key regulator that promotes $\mathrm{BC}$ growth through DMTF1 regulation.

Interestingly, our results revealed that miR-155-5p had significantly lower expression in plasma samples of patients with 
UC compared to patients with CKD. However, other reports showed that miR-155-3p and miR-155-5p had higher expression in the urine, plasma and tissues of patients with BC compared to the healthy population $(24,27,38,39)$. In addition, three studies indicated different expression levels of miR-378 (miR378-3p or miR-378-5p) in RCC compared to the serum of healthy (40-42). Importantly, the statistical methods, including the calculation of expression levels and different internal controls, led to different results. The expression levels of miRNAs were inconsistent between cells and urine, possibly due to tissue specificity or the different functional effects between cellular and extracellular environments.

Our results showed that lower expression of miR-19a (miR19a-3p or miR-19a-5p), miR-19b (miR-19b-3p or miR-19b-1-5p), miR-636 and miR-378 and higher expression of miR-708-5p were linked to the poor prognosis of patients with BC (Figure 3A). On the other hand, the group with lower miR-30a expression and higher miR-155 expression was linked to the poor prognosis of ccRCC (Figure 3B). Interestingly, a previous study showed that high miR-19a-3p expression was associated with poor prognosis of prostate cancer (43). Low expression of miR-19b-1-5p in tissue was linked to poor prognosis of BC, and low miR-19b-3p expression in patients suffering from prostate cancer also showed poor prognosis $(24,43)$. Poor prognosis was also found in the group with low miR-378 (miR-378-3p or miR-378-5p) expression in the plasma of RCC (41). In a previous study, miR-708-5p was reported in non-small cell lung cancer, ovarian cancer and stomach cancer (44-46).

\section{CONCLUSIONS}

In this study, we aimed to establish predictive models of UC using miRNA expression levels in the urine and plasma. The prediction models and nomograms could be an ancillary diagnostic marker for patients with CKD, who are at high risk of developing UC. As far as we know, this is the first study to investigate UC in CKD patients by miRNA expression levels in their biofluids.

\section{DATA AVAILABILITY STATEMENT}

The original contributions presented in the study are included in the article/Supplementary Material, further inquiries can be directed to the corresponding author/s.

\section{REFERENCES}

1. Bray F, Ferlay J, Soerjomataram I, Siegel RL, Torre LA, Jemal A. Global cancer statistics 2018: GLOBOCAN estimates of incidence and mortality worldwide for 36 cancers in 185 countries. CA Cancer J Clin. (2018) 68:394424. doi: 10.3322/caac. 21492

2. Kaseb H, Aeddula NR. Cancer, Bladder. Treasure Island, FL: StatPearls (2019).

3. Yang MH, Chen KK, Yen CC, Wang WS, Chang YH, Huang WJ, et al. Unusually high incidence of upper urinary tract urothelial carcinoma in Taiwan. Urology. (2002) 59:681-7. doi: 10.1016/S0090-4295(02)01529-7

4. Lowrance WT, Ordonez J, Udaltsova N, Russo P, Go AS. CKD and the risk of incident cancer. $J$ Am Soc Nephrol. (2014) 25:2327-34. doi: 10.1681/ASN.2013060604

\section{ETHICS STATEMENT}

The studies involving human participants were reviewed and approved by CMUH 102-REC2-043. The patients/participants provided their written informed consent to participate in this study.

\section{AUTHOR CONTRIBUTIONS}

C-CH and NM: conceptualization. A-LL, C-YC, and S-CL: methodology. A-LL, C-YC, NM, and C-CH: investigation and validation. C-LC, K-LW, H-CC, M-CW, C-CC, B-GH, and M-SW: resources. A-LL: writing-original draft. NM, C-LC, and $\mathrm{C}-\mathrm{CH}$ : review and editing. NM, C-LC, and C-CH: funding acquisition. All authors contributed to the article and approved the submitted version.

\section{FUNDING}

This work was supported by the following programs: Academia Sinica, Grant Numbers BM10701010023, BM10601010037, BM104010113, and BM103010089, NCU-Landseed International Chronic Disease Research Center, Grant Numbers NCU-LSH108-A-005 and NCU-LSH-109-A-004, Ministry of Science and Technology, Grant Numbers MOST109-2628-B-008-001 and MOST 110-2823-8-008-002, and National Health Research Institutes, Grant Number NHRI-109BCCO-MF-202018-01.

\section{ACKNOWLEDGMENTS}

We would like to thank Kuo-Hsiung Shu of Lin Shin Hospital and Yun-Ru Chiang and De-Xin Kong of National Central University and the assistants from ten hospitals for sample collection and processing. The authors thank the technical support provided by the Core Facilities for High Throughput Experimental Analysis of the Institute of Systems Biology and Bioinformatics, National Central University.

\section{SUPPLEMENTARY MATERIAL}

The Supplementary Material for this article can be found online at: https://www.frontiersin.org/articles/10.3389/fmed. 2021.726214/full\#supplementary-material

5. Chen JS, Lu CL, Huang LC, Shen CH, Chen SC. Chronic kidney disease is associated with upper tract urothelial carcinoma: a nationwide population-based cohort study in Taiwan. Medicine. (2016) 95:e3255. doi: 10.1097/MD.0000000000003255

6. Hsieh JJ, Purdue MP, Signoretti S, Swanton C, Albiges L, Schmidinger M, et al. Renal cell carcinoma. Nat Rev Dis Primers. (2017) 3:17009. doi: 10.1038/nrdp.2017.9

7. Hatakeyama S, Koie T, Narita T, Hosogoe S, Yamamoto H, Tobisawa Y, et al. Renal function outcomes and risk factors for stage $3 \mathrm{~B}$ chronic kidney disease after urinary diversion in patients with muscle invasive bladder cancer. PLoS ONE. (2016) 11:e0149544. doi: 10.1371/journal.pone.0149544

8. Kodama H, Hatakeyama S, Fujita N, Iwamura H, Anan G, Fukushi K, et al. Preoperative chronic kidney disease predicts poor oncological outcomes after 
radical nephroureterectomy in patients with upper urinary tract urothelial carcinoma. Oncotarget. (2017) 8:83183-94. doi: 10.18632/oncotarget.20554

9. Lotan Y, Elias K, Svatek RS, Bagrodia A, Nuss G, Moran B, et al. Bladder cancer screening in a high risk asymptomatic population using a point of care urine based protein tumor marker. J Urol. (2009) 182:527. doi: 10.1016/j.juro.2009.02.142

10. Xi Z, Linlin M, Ye T. Human epididymis protein 4 is a biomarker for transitional cell carcinoma in the urinary system. J Clin Lab Anal. (2009) 23:357-61. doi: 10.1002/jcla.20329

11. Manvar AM, Wallen EM, Pruthi RS, Nielsen ME. Prognostic value of CA 125 in transitional cell carcinoma of the bladder. Expert Rev Anticancer Ther. (2010) 10:1877-81. doi: 10.1586/era.10.186

12. Ahmadi H, Djaladat H, Cai J, Miranda G, Daneshmand S. Precystectomy serum levels of carbohydrate antigen 19-9, carbohydrate antigen 125, and carcinoembryonic antigen: prognostic value in invasive urothelial carcinoma of the bladder. Urol Oncol. (2014) 32:648-56. doi: 10.1016/j.urolonc.2014.01.019

13. Chou CY, Shu KH, Chen HC, Wang MC, Chang CC, Hsu BG, et al. Development and validation of a nomogram for urothelial cancer in patients with chronic kidney disease. Sci Rep. (2019) 9:3473. doi: 10.1038/s41598-019-40276-4

14. Sanli O, Dobruch J, Knowles MA, Burger M, Alemozaffar M, Nielsen ME, et al. Bladder cancer. Nat Rev Dis Primers. (2017) 3:17022. doi: 10.1038/nrdp.2017.22

15. Gaggl M, Hofer M, Weidner S, Kleinert J, Fauler G, Wallner M, et al. Interfering parameters in the determination of urinary globotriaosylceramide (Gb3) in patients with chronic kidney disease. J Nephrol. (2015) 28:67989. doi: 10.1007/s40620-015-0193-1

16. Fendler A, Stephan C, Yousef GM, Kristiansen G, Jung K. The translational potential of microRNAs as biofluid markers of urological tumours. Nat Rev Urol. (2016) 13:734-52. doi: 10.1038/nrurol.2016.193

17. Mytsyk Y, Dosenko V, Skrzypczyk MA, Borys Y, Diychuk Y, Kucher A, et al. Potential clinical applications of microRNAs as biomarkers for renal cell carcinoma. Cent European J Urol. (2018) 71:295-303. doi: 10.5173/ceju.2018.1618

18. Andersen GB, Tost J. Circulating miRNAs as biomarker in cancer. Recent Results Cancer Res. (2020) 215:277-98. doi: 10.1007/978-3-030-26439-0_15

19. Springer SU, Chen CH, Rodriguez Pena MDC, Li L, Douville C, Wang Y, et al. Non-invasive detection of urothelial cancer through the analysis of driver gene mutations and aneuploidy. Elife. (2018) 7:e32143. doi: 10.1101/2 03976

20. Blanca A, Sanchez-Gonzalez A, Requena MJ, Carrasco-Valiente J, GomezGomez E, Cheng L, et al. Expression of miR-100 and miR-138 as prognostic biomarkers in non-muscle-invasive bladder cancer. APMIS. (2019) 127:54553. doi: 10.1111/apm.12973

21. Gullu Amuran G, Tinay I, Filinte D, Ilgin C, Peker Eyuboglu I, Akkiprik M. Urinary micro-RNA expressions and protein concentrations may differentiate bladder cancer patients from healthy controls. Int Urol Nephrol. (2019) 52:461-8. doi: 10.1007/s11255-019-0 2328-6

22. Lin GB, Zhang CM, Chen XY, Wang JW, Chen S, Tang SY, et al. Identification of circulating miRNAs as novel prognostic biomarkers for bladder cancer. Math Biosci Eng. (2019) 17:834-44. doi: 10.3934/mbe.20 20044

23. Usuba W, Urabe F, Yamamoto Y, Matsuzaki J, Sasaki H, Ichikawa M, et al. Circulating miRNA panels for specific and early detection in bladder cancer. Cancer Sci. (2019) 110:408-19. doi: 10.1111/cas.13856

24. Chen CL, Lin CH, Li AL, Huang CC, Shen BY, Chiang YR, et al. Plasma miRNA profile is a biomarker associated with urothelial carcinoma in chronic hemodialysis patients. Am J Physiol Renal Physiol. (2019) 316:F1094102. doi: 10.1152/ajprenal.00014.2019

25. Li AL, Chung TS, Chan YN, Chen CL, Lin SC, Chiang YR, et al. microRNA expression pattern as an ancillary prognostic signature for radiotherapy. J Transl Med. (2018) 16:341. doi: 10.1186/s12967-018-1 711-4

26. Jiang X, Du L, Wang L, Li J, Liu Y, Zheng G, et al. Serum microRNA expression signatures identified from genome-wide microRNA profiling serve as novel noninvasive biomarkers for diagnosis and recurrence of bladder cancer. Int $J$ Cancer. (2015) 136:854-62. doi: 10.1002/ijc.29041

27. Wang G, Chan ES, Kwan BC, Li PK, Yip SK, Szeto CC, et al. Expression of microRNAs in the urine of patients with bladder cancer. Clin Genitourin Cancer. (2012) 10:106-13. doi: 10.1016/j.clgc.2012. 01.001

28. Feng Y, Liu J, Kang Y, He Y, Liang B, Yang P, et al. miR-19a acts as an oncogenic microRNA and is up-regulated in bladder cancer. J Exp Clin Cancer Res. (2014) 33:67. doi: 10.1186/s13046-014-0067-8

29. Du M, Shi D, Yuan L, Li P, Chu H, Qin C, et al. Circulating miR-497 and miR$663 \mathrm{~b}$ in plasma are potential novel biomarkers for bladder cancer. Sci Rep. (2015) 5:10437. doi: 10.1038/srep10437

30. Long JD, Sullivan TB, Humphrey J, Logvinenko T, Summerhayes KA, Kozinn $\mathrm{S}$, et al. A non-invasive miRNA based assay to detect bladder cancer in cell-free urine. Am J Transl Res. (2015) 7:2500-9.

31. Song T, Zhang X, Zhang L, Dong J, Cai W, Gao J, et al. miR-708 promotes the development of bladder carcinoma via direct repression of Caspase2. J Cancer Res Clin Oncol. (2013) 139:1189-98. doi: 10.1007/s00432-013-1 392-6

32. Zhang C, Ma X, Du J, Yao Z, Shi T, Ai Q, et al. MicroRNA-30a as a prognostic factor in urothelial carcinoma of bladder inhibits cellular malignancy by antagonising Notch1. BJU Int. (2016) 118:578-89. doi: 10.1111/bju. 13407

33. Chung YH, Li SC, Kao YH, Luo HL, Cheng YT, Lin PR, et al. MiR-30a-5p inhibits epithelial-to-mesenchymal transition and upregulates expression of tight junction protein claudin-5 in human upper tract urothelial carcinoma cells. Int J Mol Sci. (2017) 18:1826. doi: 10.3390/ijms18081826

34. Yoshino H, Yonezawa T, Yonemori M, Miyamoto K, Sakaguchi T, Sugita S, et al. Downregulation of microRNA-1274a induces cell apoptosis through regulation of BMPR1B in clear cell renal cell carcinoma. Oncol Rep. (2018) 39:173-81. doi: 10.3892/or.2017.6098

35. Niu S, Ma X, Zhang Y, Liu YN, Chen X, Gong H, et al. MicroRNA$19 \mathrm{a}$ and microRNA-19b promote the malignancy of clear cell renal cell carcinoma through targeting the tumor suppressor RhoB. PLoS ONE. (2018) 13:e0192790. doi: 10.1371/journal.pone.01 92790

36. Yin $\mathrm{XH}$, Jin $\mathrm{YH}$, Cao $\mathrm{Y}$, Wong $\mathrm{Y}$, Weng H, Sun C, et al. Development of a 21-miRNA signature associated with the prognosis of patients with bladder cancer. Front Oncol. (2019) 9:729. doi: 10.3389/fonc.2019. 00729

37. Nguyen HC, Xie W, Yang M, Hsieh CL, Drouin S, Lee GS, et al. Expression differences of circulating microRNAs in metastatic castration resistant prostate cancer and low-risk, localized prostate cancer. Prostate. (2013) 73:346-54. doi: 10.1002/pros.22572

38. Peng Y, Dong W, Lin TX, Zhong GZ, Liao B, Wang B, et al. MicroRNA-155 promotes bladder cancer growth by repressing the tumor suppressor DMTF1. Oncotarget. (2015) 6:16043-58. doi: 10.18632/oncotarget.3755

39. Zhang X, Zhang Y, Liu X, Fang A, Wang J, Yang Y, et al. Direct quantitative detection for cell-free miR-155 in urine: a potential role in diagnosis and prognosis for non-muscle invasive bladder cancer. Oncotarget. (2016) 7:325566. doi: 10.18632/oncotarget.6487

40. Redova M, Poprach A, Nekvindova J, Iliev R, Radova L, Lakomy R, et al. Circulating miR-378 and miR-451 in serum are potential biomarkers for renal cell carcinoma. J Transl Med. (2012) 10:55. doi: 10.1186/1479-587 6-10-55

41. Fedorko M, Stanik M, Iliev R, Redova-Lojova M, Machackova T, Svoboda $\mathrm{M}$, et al. Combination of MiR-378 and MiR-210 serum levels enables sensitive detection of renal cell carcinoma. Int J Mol Sci. (2015) 16:233829. doi: 10.3390/ijms161023382

42. Wang C, Hu J, Lu M, Gu H, Zhou X, Chen X, et al. A panel of five serum miRNAs as a potential diagnostic tool for early-stage renal cell carcinoma. Sci Rep. (2015) 5:7610. doi: 10.1038/srep07610

43. Stuopelyte K, Daniunaite K, Jankevicius F, Jarmalaite S. Detection of miRNAs in urine of prostate cancer patients. Medicina. (2016) 52:11624. doi: 10.1016/j.medici.2016.02.007

44. Jang JS, Jeon HS, Sun Z, Aubry MC, Tang H, Park CH, et al. Increased miR-708 expression in NSCLC and its association with poor survival in 
lung adenocarcinoma from never smokers. Clin Cancer Res. (2012) 18:365867. doi: 10.1158/1078-0432.CCR-11-2857

45. Lin KT, Yeh YM, Chuang CM, Yang SY, Chang JW, Sun SP, et al. Glucocorticoids mediate induction of microRNA708 to suppress ovarian cancer metastasis through targeting Rap1B. Nat Commun. (2015) 6:5917. doi: 10.1038/ ncomms6917

46. Liang L, Zhang L, Cui D, Yang D. Identification of the key miRNAs associated with survival time in stomach adenocarcinoma. Oncol Lett. (2017) 14:456372. doi: $10.3892 /$ ol.2017.6792

Conflict of Interest: The authors declare that the research was conducted in the absence of any commercial or financial relationships that could be construed as a potential conflict of interest.
Publisher's Note: All claims expressed in this article are solely those of the authors and do not necessarily represent those of their affiliated organizations, or those of the publisher, the editors and the reviewers. Any product that may be evaluated in this article, or claim that may be made by its manufacturer, is not guaranteed or endorsed by the publisher.

Copyright (c) $2021 \mathrm{Li}$, Chou, Chen, Wu, Lin, Chen, Wang, Chang, Hsu, Wu, Ma and Huang. This is an open-access article distributed under the terms of the Creative Commons Attribution License (CC BY). The use, distribution or reproduction in other forums is permitted, provided the original author(s) and the copyright owner(s) are credited and that the original publication in this journal is cited, in accordance with accepted academic practice. No use, distribution or reproduction is permitted which does not comply with these terms. 